\title{
Influences of Processing Technique on Electrical Characteristics of TVS used in Communication Systems
}

\author{
X. R. Zheng, B.Y.Liu, Bin Li and P.T. Lai \\ Department of Electrical and Electronic Engineering, the University of Hong Kong \\ (E-Mail:laip@hkueee.hku.hk)
}

Area: Silicon Devices and Technology

\begin{abstract}
An improved technique is proposed in fabricating a semiconductor surge protection device which is used in high-speed wideband information transmission systems. In order to increase the surge handling capability of the device, a double p-type diffusion is used. Specifically, in the diffusion step of gallium, $\mathrm{SiO}_{2}$ is used as a mask to obtain a very small base width and to avoid the reduction of carrier lifetime. It is found that this is a very useful way to reduce the on-state voltage drop and therefore the energy dissipation of the device.
\end{abstract}

\section{INTRODUCTION}

Nowadays, electronics are increasingly based on digital signals. Digital systems are providing more and more advanced electronic communication services (simultaneous voice and data transfer, screenphone, message handling, etc.). The growing integration of functions makes these systems operate at a wider working band and higher transfer rate, and the energy required for information transfer has been considerably reduced. Furthermore, these systems are mainly made up of VLSI's which are very vulnerable to electromagnetic interference, such as lightning stroke, industrial or operating transient overvoltage. Therefore, the safety and protection of these systems are very important. The protection semiconductor devices used in these systems are generally named as transient voltage suppressor (TVS), whose requirements are summarized as :

1. high surge current handling capability;

2. fast response to the surge and low device capacitance to reduce transmission loss in high-speed wideband transmission systems;
3. high holding current, ensuring the device can be turned off when a surge current rapidly decays;

4. proper protection voltage (i.e. forward breakover voltage), whose exact value is predetermined by the detailed structure of the device;

5. very low leakage current.

A bi-directional TVS is shown in Figure 1(a). It is similar to un-gated pnpn switches which are designed to switch from off-state to on-state when a specified voltage or $\mathrm{dV} / \mathrm{dt}$ is reached, as described by the I-V characteristic of TVS in Fig.1(b). Since the influences of device area on both the surge capability and the device capacitance are conflicting, the basic consideration of TVS design lies on a small device area adopted to keep device capacitance low enough, and increasing the per-unit-area surge handling capability, which implies that the energy dissipation and temperature rise in the device must be greatly decreased when a surge is induced. On-state voltage drop. is a key parameter for the requirement of low energy dissipation. This paper presents an improved fabrication process for decreasing the on-state voltage drop of TVS.

\section{THEORETICAL CONSIDERATION}

\section{Device capacitance}

From Fig.1(a), device capacitance $C_{\text {TVS }}$

$$
\frac{1}{C_{T V S}}=\frac{1}{C_{j 1}}+\frac{1}{C_{j 2}}+\frac{1}{C_{j 3}}
$$

$C_{\mathrm{j} 2}$ is the single-side linearly graded junction capacitance under reverse bias ${ }^{[1]}$ 


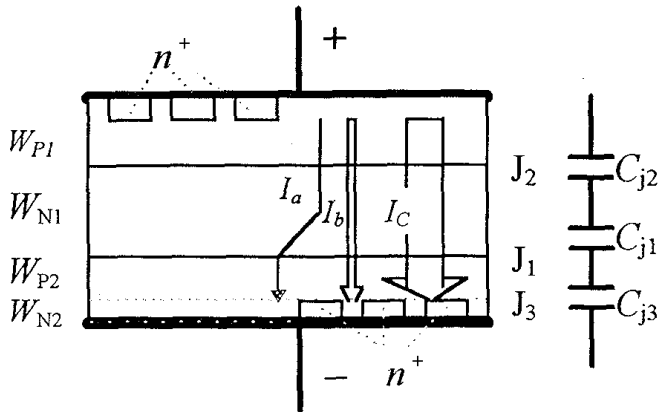

(a)

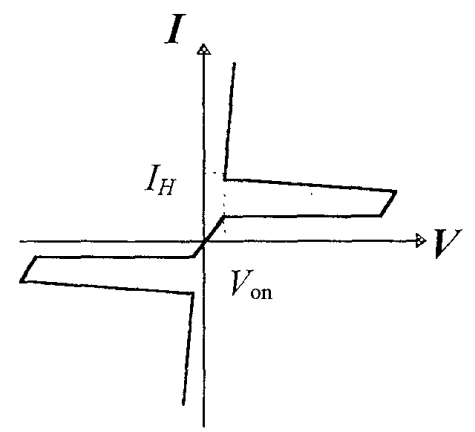

(b)

Fig. 1 (a) Cross-sectional view and (b) typical I-V characteristic of a bi-directional TVS

$$
C_{j 2}=\left[\frac{q \varepsilon N_{N 1}}{2\left(V_{b i}+V-2 k T / q\right)}\right]^{1 / 2}\left(\mathrm{pF} / \mathrm{cm}^{2}\right)
$$

where $\mathrm{N}_{\mathrm{N} 1}$ is the doping concentration of the $\mathrm{N}_{1}$ layer; $V_{\mathrm{bi}}$ is the built-in potential of the pn junction; $V$ is the applied voltage. $C_{\mathrm{j} 1}, C_{\mathrm{j} z}$ are the depletion capacitance plus diffusion capacitance of the other two pn junctions under forward bias. Since $C_{\mathrm{j} 1} \gg C_{\mathrm{j} 2}$ and $C_{\mathrm{j} 3}$ $\gg C_{\mathrm{j} 2}, C_{\mathrm{rvS}} \approx C_{\mathrm{j} 2}$. When $\mathrm{N}_{\mathrm{N} 1}=7.3 \times 10^{14} / \mathrm{cm}^{3}$, $C_{\mathrm{j} 2}=4.2 \times 10^{3} \mathrm{pF} / \mathrm{cm}^{2}$ at $\mathrm{V}=0 \mathrm{~V}$. Hence, for a device area of $0.21 \times 0.21 \mathrm{~cm}^{2}, C_{\text {TVS }}$ is $185 \mathrm{pF}$ at $\mathrm{V}=0 \mathrm{~V}$, and $C_{\mathrm{TVs}}=79 \mathrm{pF}$ at $\mathrm{V}=40 \mathrm{~V}$. These values satisfy the requirement of the TVS used in communication systems ${ }^{[2]}$.
Surge capability, i.e. the ability of a device conducting a large transient surge current without being damaged, mainly depends on the total energy dissipation $E$ in the device during an induced surge which can be expressed as in equation (3), where $E_{\mathrm{a}}$ is the energy dissipation related to the avalanche current $I_{\mathrm{a}} ; \quad E_{\mathrm{b}}$ and $E_{\mathrm{on}}$ are the energy dissipations of the device in turn-on and onstate periods respectively. Since the period of $t_{3}-t_{4}$ is much longer than $t_{1}-t_{3}$ in (3), $E_{\text {on }}$ dominates $E$. Therefore, the reduction of onstate voltage drop $V_{\text {on }}$ is a key for the reduction of $E$.

Under high-injection level, $V_{\text {on }}$ is given by ${ }^{[3]}$ equation (4), where $L_{\mathrm{a}}, D_{\mathrm{a}}$ and $\tau_{\mathrm{a}}$ are the diffusion length, mobility and lifetime of the charge carriers respectively. $\mathrm{W}_{\mathrm{N}_{2}}$ and $\mathrm{N}_{\mathrm{N} 2}$ are the width and doping concentration of the $\mathrm{N}_{2}$ layer. Base width $\mathrm{W}_{\mathrm{T}}=\mathrm{W}_{\mathrm{P} 1}+\mathrm{W}_{\mathrm{N} 1}+\mathrm{W}_{\mathrm{P} 2}$, as shown in Fig.1. $R_{\mathrm{B}}$ and $R_{\text {CoNT }}$ are bulk resistance and ohmic contact resistance of the device. Equation (4) shows that $V_{\text {on }}$ is a very strong function of $\mathrm{W}_{\mathrm{T}}$, approximating to a square-law dependence and is inversely proportional to the square root of the carrier lifetime.

\section{EXPERIMENTAL RESULTS AND DISCUSSIONS}

\section{Sample preparation}

The TVS was fabricated mainly by two steps of p-type and n-type impurity diffusions. The electrical characteristics of the device such as breakover forward voltage, on-state voltage drop, holding current are dependent on the first p-type diffusion step which results

\section{On-state voltage drop}

$$
\begin{aligned}
& E=E_{a}+E_{b}+E_{o n}=\int_{t_{1}}^{t_{2}} V_{a}(t) I_{a}(t) d t+\int_{t_{2}}^{t_{3}} V_{b}(t) I_{b}(t) d t+\int_{t_{3}}^{t_{4}} V_{o n}(t) I_{C}(t) d t \\
& V_{o n}=\frac{k T}{q} \ln \left(\frac{W_{N 2} N_{N 2}}{2 q D_{a} n_{i}^{2}} J\right)+\frac{W_{T}^{2}}{2 \mu \tau_{a}^{1 / 2}} \frac{\sinh \left(W_{T} / L_{a}\right)}{\left[\cosh \left(W_{T} / L_{a}\right)-1\right]\left(2 q W_{N 2} N_{N 2}\right)^{1 / 2}}+\left(R_{B}+R_{C O N T}\right) \cdot I_{C}
\end{aligned}
$$


Table I p-type diffusion methods

\begin{tabular}{|c|c|c|c|}
\hline method & I & II & \multicolumn{1}{c|}{ III } \\
\hline & $\begin{array}{c}\text { boron-aluminium coated } \\
\text { source sealed-tube }\end{array}$ & $\begin{array}{c}\text { aluminium-gallium } \\
\text { sealed-tube }\end{array}$ & $\begin{array}{c}\text { aluminium-gallium } \\
\text { sealed-tube }\end{array}$ \\
\hline Group A & $1250^{\circ} \mathrm{C}$ & $1250^{\circ} \mathrm{C}$ \\
& $38 \sim 45 \mathrm{~h}$ & $\begin{array}{l}\text { Same as method II } \\
\text { except } 0.8 \mu \mathrm{mm} \mathrm{SiO} \text { used } \\
\text { as mask during Ga } \\
\text { diffusion }\end{array}$ \\
\hline Group B & $\begin{array}{c}32 \mathrm{~h}(\mathrm{Al})+4 \mathrm{~h}(\mathrm{Ga}) \\
+8 \mathrm{~h}(\text { drive in) }\end{array}$ & $\begin{array}{c}1250^{\circ} \mathrm{C} \\
24 \mathrm{~h}(\mathrm{Al})+4 \mathrm{~h}(\mathrm{Ga}) \\
+4 \mathrm{~h}(\text { drive in) }\end{array}$ & \\
\hline
\end{tabular}

in an optimum junction depth, impurity profile and carrier lifetime. The choice of diffusion technique is decisive to achieving the required characteristics. The present experiment used three p-type diffusion methods with two diffusion times in each case. The details of the sample preparation are summarized in Table I. These methods are all double impurity diffusion, i.e. a deep diffusion of aluminum with low concentration followed by a shallower diffusion of gallium or boron with high concentration. The resulting doping profile is sketched in Fig.2. The size of the samples is $0.044 \mathrm{~cm}^{2}$, and the original thickness of silicon chip is $0.26 \mathrm{~mm}$.

\section{Results and discussions}

The I-V characteristics of the samples are measured and their $V_{\text {on }}$ and $I_{\mathrm{H}}$ are plotted in Fig. 3. It is clear that $V_{\text {on }}$ of group B is greater than that of group A, as predicted in (4). It is particularly meaningful that the samples made by method III have a slightly lower $V_{\text {on }}$ than those by method II. This phenomenon is probably due to the higher carrier lifetime resulting from the diffusion III. Gallium doping source or the processing system may be contaminated by heavy metal species, such as copper, iron, gold, etc, which introduce at least two impurity levels in the band gap that act as the recombination centers and result in a shorter hole lifetime in the n-base. These heavy metal elements, are fast diffusing elements in silicon, but have a very low diffusion coefficient in $\mathrm{SiO}_{2}{ }^{[4]}$. On the other hand, the diffusion coefficient of gallium in $\mathrm{SiO}_{2}$ is very high (about $8 \times 10^{-9} \mathrm{~cm}^{2} / \mathrm{s}$ at 1200
${ }^{\circ} \mathrm{C}$ ), and over 1000 times larger than those of the heavy metal contaminants. In other words, $\mathrm{SiO}_{2}$ is transparent to gallium but is a mask for the heavy metal contaminants at high temperatures. In method $\mathrm{III}, \mathrm{SiO}_{2}$ resists the heavy metal contaminants into silicon, and avoids the reduction of carrier lifetime. A lower $V_{\text {on }}$ can therefore be obtained.

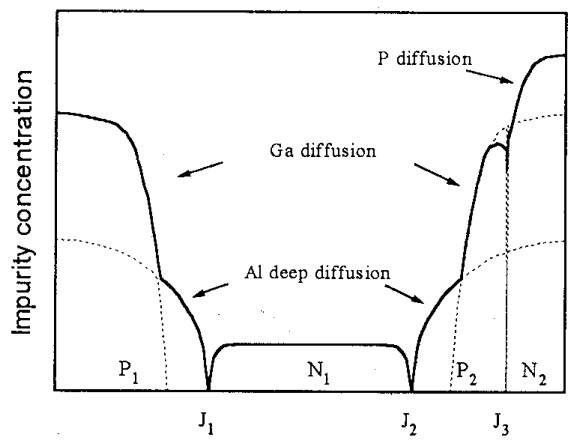

Fig.2 Doping profile of the TVS device with double impurity diffusion

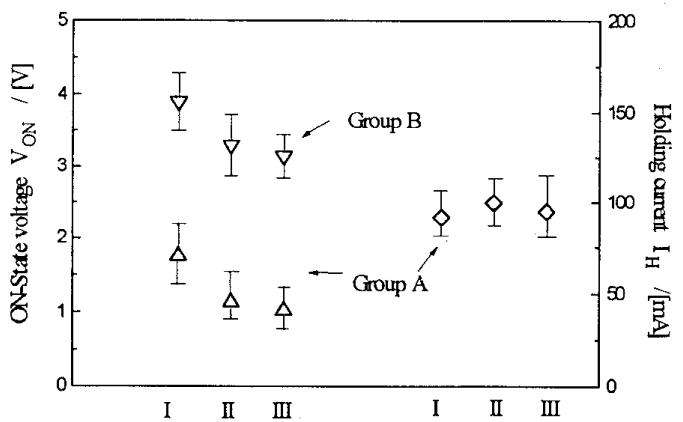

Fig.3 On-state voltage (triangles) and holding current (diamonds) resulting from different diffusion methods

A theoretical I-V characteristic of a pnpn structure was derived by Kurata ${ }^{[5]}$ by solving a complete set of semiconductor device equations. It was shown that the holding 
current was reduced by a factor of 100 as the lifetime increased from 0.8 to $2 \mu$ s. However, the holding current remains almost the same in our experiment. This is probably due to the existence of cathode shorting which dominates the value of holding current.

\section{CONCLUSION}

Three types of p-type diffusion methods are studied to clarify the dependence of the surge capability of TVS on base width and carrier lifetime by measuring the on-state voltage drop of the device. Aluminum and gallium double diffusion can produce a very narrow base. Furthermore, a layer of $\mathrm{SiO}_{2}$ is used as a mask against heavy metal contaminants in the gallium diffusion step to avoid the degradation of carrier lifetime and to decrease the on-state voltage drop further.

\section{ACKNOWLEDGMENTS}

The project is partially supported by Natural Science Foundation of Guangdong Province of China, the CRCG Grant of the University of Hong Kong. The authors wish to express their gratitude for the help in making the samples to the Semiconductor Factory of The Research Institute of Electric Locomotive of Zhuzhou, under State Railway Ministries of China. The authors are greatly indebted to the warm help of Mr. C.L.Chan at IC/Thin Film Lab. of HKU.

\section{References}

[1] S. M. Sze, Physics of Semiconductor Devices. New York:Wiley,1981, ch.2

[2] The International Telegraph and Telephone Consultative Committee, Recommendation (CCITT), K.28, Geneva, 1991.

[3] Paul D. Taylor, Thyristor Design and Realization. New York: John Wiley \& Sons, P62.

[4] H. F. Wolf, Silicon semiconductor Data. Signetics corporation, New York, 1969.
[5] M. Kurata, One-dimensional calculation of thyristor forward voltage and holding currents, Solid State Electronics, 1976, Vol.19, pp 527-535. 
A Predictive Semi-Analytical Threshold Voltage Model for DeepSubmicron MOSFET's

K. Y. Lim 1 , X. Zhou', D. Lim², Y. Zu², H. M. Ho ${ }^{2}$, K. Loiko' ${ }^{2}$ C. K. Lau²,

M. S. Tse ${ }^{1}$, and S. C. Choo ${ }^{1},{ }^{1}$ Nanyang Technology University,

Singapore, ${ }^{2}$ Chartered Semiconductor Manufacturing Ltd., Singapore

Modeling for the Subthreshold Current in SOI Short Channel Gate Controlled Hybrid Transistor

R. Huang, Y. Y. Wang, and R. Q. Han, Peking University, P. R. China

A New Approach to Study the Channel Charge Partition of MOS Transistors During Non-Quasitic (NQS) Turn-On

W.-K. Lee, and M. Chan, Hong Kong University of Science \& Technology, Hong Kong

Modeling of Electromigration-Induced Resistance Change in Aluminum Thin Films

V. C. Lo, Hong Kong Polytechnic University, Hong Kong

Negative Binomial Yield Model Parameter Extraction Using Wafer Probe Bin Map Data

R. E. Langford ${ }^{1,2}$, and J. J. Liou' ${ }^{2}$, ${ }^{1}$ Lucent Technologies, U.S.A., ${ }^{2}$ Univ. of Central Florida, U.S.A.

The Parallel Simulation Software Design Method On Hydrodynamic Model

L. Yang, J. Wu, Nasirjan, T. Wei, Y. Wang, J. Xie, and M. Cai, Southeast University, P. R. China 